\title{
A rapid near-patient RT-PCR test for suspected COVID-19: a study of the diagnostic accuracy
}

\author{
Paul Hofman ${ }^{1,2,3}$, Jacques Boutros ${ }^{4}$, Didier Benchetrit ${ }^{5}$, Jonathan Benzaquen ${ }^{3,4}$, Sylvie Leroy ${ }^{4,6}$, \\ Virginie Tanga $^{1,2}$, Olivier Bordone ${ }^{1,2}$, Maryline Allégra ${ }^{1,2}$, Virginie Lespinet ${ }^{1,2}$, Julien Fayada ${ }^{1,2}$, \\ Charlotte Maniel $^{4}$, Jennifer Griffonnet ${ }^{4}$, Eric Selva ${ }^{1,2}$, Giancarlo Troncone ${ }^{7}$, Giuseppe Portella ${ }^{7}$, \\ Thibaut Lavrut $^{8}$, Richard Chemla ${ }^{9}$, Michel Carles ${ }^{10}$, Marius Ilié ${ }^{1,2,3}$, Charles Marquette
}

${ }^{1}$ Laboratory of Clinical and Experimental Pathology, FHU OncoAge, Centre Hospitalier Universitaire de Nice, Université Côte d'Azur, Nice, France; ${ }^{2}$ Université Côte d'Azur, FHU OncoAge, Centre Hospitalier Universitaire de Nice, Hospital-Related Biobank (BB-0033-00025), Nice, France; ${ }^{3}$ Institute of Research on Cancer and Aging (IRCAN), Université Côte d'Azur, CNRS, INSERM, Nice, France; ${ }^{4}$ Department of Pulmonary Medicine and Oncology, Université Côte d'Azur, Centre Hospitalier Universitaire de Nice, FHU OncoAge, Nice, France; ${ }^{5}$ SYNLAB Barla, 41 boulevard Louis Braille, Nice, France; ${ }^{6}$ CNRS UMR 7275 - Institut de Pharmacologie Moléculaire et Cellulaire - Université Côte d'Azur, Nice, France; ${ }^{7}$ Department of Public Health, University of Naples Frederico II, Naples, Italy; ${ }^{8}$ Laboratoire de Virologie CHU de Nice, Université Côte d'Azur, Centre Hospitalier Universitaire de Nice, Nice, France; ${ }^{9}$ Ville de Nice, Nice, France; ${ }^{10}$ Department of Infectious Diseases, Université Côte d'Azur, Centre Hopsitalier Universitaire de Nice, Nice, France

Contributions: (I) Conception and design: P Hofman, S Leroy, C Marquette; (II) Administrative support: V Tanga, O Bordone, M Allégra, V Lespinet, J Fayada, C Maniel, J Griffonnet; (III) Provision of study materials or patients: P Hofman, R Chemla, G Troncone, G Portella, T Lavrut, M Carles, M Ilié; (IV) Collection and assembly of data: C Maniel, C Marquette, J Boutros; (V) Data analysis and interpretation: J Benzaquen; (VI) Manuscript writing: All authors; (VII) Final approval of manuscript: All authors.

Correspondence to: Paul Hofman. Laboratory of Clinical and Experimental Pathology, Centre Hospitalier Universitaire de Nice, Hôpital Pasteur, 30 avenue de la voie romaine, 06001 Nice, France. Email: hofman.p@chu-nice.fr.

Background: Management of large numbers of reverse transcriptase-polymerase chain reactions (RT-PCR) for diagnosis of coronavirus 2019 disease (COVID-19) requires robust infrastructures, located in dedicated premises with a high standard of biosafety procedures, and well-trained personnel. The handling of a "runof-river sample" to obtain rapid reporting of results is challenging.

Methods: We studied the clinical performance of the Idylla ${ }^{\mathrm{TM}}$ SARS-CoV-2 Test (index test) on a platform capable of fully automated nucleic acid testing including extraction, amplification, and detection in a singleuse cartridge to establish the diagnosis of COVID-19. The study was conducted on a prospective cohort of 112 volunteers with recent symptoms and an unknown SARS-CoV-2 status who came to free screening centers of the Nice metropolitan area. All subjects underwent bilateral nasopharyngeal sampling. One sample was processed using the index test, the other using the standard of care RT-PCR. Samples were treated blind. Results: Most of the participants (70\%) were sampled within 4 days of symptom onset. Forty-five (40.2\%) were positive for COVID-19. No clinical symptoms were distinguished between SARS-CoV-2 RT-PCR positive and negative subjects except anosmia and dysgeusia. Positive and negative agreement between the index and the standard of care test was $100 \%$.

Conclusions: The Idylla ${ }^{\mathrm{TM}}$ SARS-CoV-2 Test is very sensitive, specific, rapid and easy to use in a nearpatient RT-PCR approach to distinguish between symptomatic SARS-CoV-2 positive and negative patients in selected settings.

Keywords: Coronavirus 2019 disease (COVID-19); SARS-CoV-2; reverse transcriptase-polymerase chain reactions (RT-PCR); diagnosis

Submitted Feb 10, 2021. Accepted for publication Mar 21, 2021.

doi: $10.21037 /$ atm-21-690

View this article at: http://dx.doi.org/10.21037/atm-21-690 


\section{Introduction}

So far, reverse transcriptase-polymerase chain reaction (RTPCR) testing of naso-pharyngeal swab (NPS) specimens is the gold standard for diagnosis of coronavirus 2019 disease (COVID-19) (1). In a pandemic setting, the management of hundreds or even thousands of laboratory tests in daily practice requires robust infrastructures, in dedicated premises with high standards of biosafety, as well as an adapted number of well-trained health care workers (HCWs) (2). The RT-PCR laboratory most often requires that several dozen or even hundreds of samples be grouped together at the same time in batches to be tested in parallel. The results are obtained 6-24 hours after taking the NPS but may be even longer depending on the local conditions and organization of sample workflow and technologies. So, the handling of a "run-of-river sample" giving rapid result reporting can be challenging, if not impossible in numerous situations. In routine clinical practice there are many situations where rapid individual management of an NPS for a RT-PCR SARS-CoV-2 diagnosis may be of strong interest and so an urgent need. Therefore, a nonexhaustive list of examples could include: (I) the urgent need for the diagnosis of severe pneumonia of unknown origin in a context of low prevalence of COVID-19, (II) the characterization of the COVID-19 status of a passenger before a plane flight, (III) the characterization of the COVID-19 status before emergency surgery, vaginal delivery or caesarean section, (IV) the presence of HCWs performing mandatory tasks to sustain the clinical management of patients, notably in some emergency units or for laboratory testing. In these situations, there is an urgent need for a rapid, robust, sensitive and specific near-patient test, which should be performed under environmental conditions that minimize the biohazard risk. Moreover, since the HCWs require only a short period of training to practice the test, he/she can be immediately operational. No specific expertise for handling samples, such as centrifugation, nucleic acids extraction, dilution procedures under a hood or manipulating equipment for molecular biology analyses is required.

The purpose of this study was to compare the clinical performance of a new and rapid RT-PCR test for SARSCoV-2 detection (Idylla ${ }^{\mathrm{TM}}$, Biocartis NV, Mechelen, Belgium) with NPS taken from symptomatic patients with the SOC methods for SARS-CoV-2 detection that are already set up in the Nice metropolitan area (France). The Idylla ${ }^{\mathrm{TM}}$ SARS-CoV-2 Test is an automated RT-PCR test intended for the qualitative detection of RNA from the SARS-CoV-2 in nasopharyngeal swab specimens from individuals suspected of COVID-19. The Idylla ${ }^{\mathrm{TM}}$ SARSCoV-2 Test Cartridges are ready-for-use and contain the necessary reagents to perform sample preparation, cell lysis and extraction, reverse transcription, PCR amplification and high-resolution detection, starting from insertion of Viral Transport Media (VTM) specimens. In Idylla, 5 PCR reactions take place, 3 identical $O R F 1 b$ and 2 identical $N$ reactions, each using about $1 / 10$ of the extracted nucleic acids. So in total, about half of the sample equivalent in terms of target nucleic acids is used from the $200 \mu \mathrm{L}$ VTM sample, which may strongly increase the chance of detection and improves test sensitivity in comparison to the test used for the SOC which develops only 2 PCR reactions taking place on $1 O R F 1 b$ and $1 \mathrm{~N}$. We present the following article in accordance with the MDAR reporting checklist (available at http://dx.doi.org/10.21037/atm-21-690).

\section{Methods}

\section{Study oversight and study population}

A prospective cohort study was conducted in consecutive adult volunteers with an unknown SARS-CoV-2 status in: (I) downtown free screening centres available to the population of the Nice metropolitan area and, (II) the outpatient clinic of the Department of Pulmonary Medicine of the University Hospital (CHU) of Nice. Participants were recruited on a voluntary basis after signing a written informed consent. To participate, volunteers had to present at least two recent ( $\leq 2$ weeks) common symptoms of COVID-19 (3). All participants were examined and medically interrogated by the same physician (CHM).

\section{Diagnostic techniques}

All participants underwent bilateral NPS by the same operator (CHM), with an ultra-thin sterile disposable nylon sampling swab (type: A-04. Jiangsu Han-Heng Medical technology Co., Ltd. Changzhou, Jiangsu, China). One swab was transported within 4 hours in $400 \mu \mathrm{L}$ of medium (DA0940. Da An Gene Co., Ltd. of Sun Yat-sen University. Guangzhou, Guangdong, China) to the SYNLAB Barla laboratory (Nice, France) or in $3 \mathrm{~mL}$ of medium (Remel MicroTest ${ }^{\mathrm{TM}}$ M4RT ${ }^{\circledR}$ Lenexa, KS, USA) to the Laboratory of Virology of the CHU of Nice, depending on where the screening tests were routinely performed. The other NPS 
was transported within no more 4 hours in $3 \mathrm{~mL}$ of medium (Remel MicroTest ${ }^{\mathrm{TM}}$ M4RT ${ }^{\circledR}$ ) to the COVID-19 Biobank of the Laboratory of Clinical and Experimental Pathology, Pavilion I, Pasteur 1 Hospital, Nice, France) (4). RTPCR testing was performed upon arrival in the respective laboratories. All these laboratories are accredited according to the ISO 15189 standard for performance of biological and molecular analyses (www.cofrac.fr). The different analyses were processed in a double-blind way.

\section{RT-PCR testing used as the SOC methods}

At the SYNLAB Barla reference laboratory the NPS were tested using the DAAgene kit (NE/CA09/170/D01/ IVD/016-03) (Da An Gene Co., Ltd. of Sun Yat-sen University, Guangzhou, Guangdong, China) as described in the instructions from the manufacturer, and processed on a AGS 4800 Thermocycler (Hangzhou, Zhejiang, China). The test includes 2 genes $(N$ and $O r f 1 b)$ Three primers per gene were used as follows:

ORF 1: (F): CCCTGTGGGTTTTACACTTAA, (R): ACGATTGTGCATCAGCTGA, (P): 5'-FAMCCGTCTGCGGTATGTGGAAAGGTTATGGBHQ1-3'

N: (F): GGGGAACTTCTCCTGCTAGAAT, (R): CAGACATTTTGCTCTCAAGCTG, (P): 5'-FAMTTGCTGCTGCTTGACAGATT-TAMRA-3'.

At the Laboratory of Virology of the CHU of Nice the NPS were tested with the SARS-CoV-2R gene test (Biomérieux, Marcy-l'Etoile, France) on an Applied Biosystems Instrument (7500 Fast Real-time PCR System, ThermoFisher Scientific, Paris, France). The results were available to the clinician within $8 \pm 24 \mathrm{~h}$ and were considered as positive when the CT (cycle threshold) value for the $\mathrm{N}$ and/or on Orf1 genes was equal or less than 36.

\section{Rapid on-site RT-PCR testing on the Idylla platform for SARS-CoV2 detection}

NPS in medium were processed immediately using the following steps: after homogenization by vortexing the liquid for 30 seconds, $200 \mu \mathrm{L}$ of the liquid present in transported tubes containing the swabs were pipetted with a sterile tip (D. Dutscher, reference 134000) and placed individually into Idylla SARS-CoV-2 test cartridges (Biocartis, reference A1042/6 and A1043/6) under a BSC-2 hood (Figure S1). The Idylla platform located in the laboratory of clinical and experimental pathology is accredited according to the ISO 15189 norm for molecular biology (www.cofrac.fr). Since the Idylla ${ }^{\mathrm{TM}}$ platform set up in this laboratory contained 4 devices (Figure S2), 4 runs could be performed at the same time. So, the Idylla ${ }^{\mathrm{TM}}$ SARS-CoV-2 IUO (52 samples) and subsequently the Idylla TM SARS-CoV-2 CE-IVD (60 samples) tests (Biocartis, references A1042/6 and A1043/6, respectively) were performed on the platform with fully automated nucleic acid testing including extraction, amplification, and detection in a single-use cartridge. The duration of the run took 90 minutes $(+/-10 \mathrm{~min})$ and the report was immediately available to the biologist for transmission to the physician at distance via a modem interface. When more than 4 tubes containing NPS were registered at the same time, the additional samples were kept at $4{ }^{\circ} \mathrm{C}$ in the COVID-19 Biobank (4) before processing the test on the Idylla ${ }^{\mathrm{TM}}$ platform. Finally, after processing, the residual volumes of media were immediately aliquoted and stored at $-80{ }^{\circ} \mathrm{C}$ in the COVID-19 Biobank (4). According to the manufacturer, the Idylla ${ }^{\mathrm{TM}}$ SARS-CoV-2 Test provides a qualitative result for the presence or absence of SARSCoV-2 RNA and a Quality Status for each test. The SARS$\mathrm{CoV}-2$ test from Biocartis includes 2 genes ( $N$ and Orf1b) covered by 5 PCR targets ( $2 \mathrm{~N}$ targets and 3 Orf1b targets). A positive or a negative result was considered according to the instructions for use of the Idylla ${ }^{\mathrm{TM}}$ SARS-CoV-2 Test (BCT T013812) (https://www.biocartis.com/en/securearea). $\mathrm{Cq}$, for cycle of quantification, is the term used in the result report and official documentation of Idylla ${ }^{\mathrm{TM}}$, hence it is used when referring to Idylla ${ }^{\mathrm{TM}}$ data. A positive result requires at least $2 \mathrm{~N}$ amplified targets [by setting a cutoff on 41.9 (Cq value)] and/or at least one or more Orf1b amplified targets. Since Orf1b is highly specific, no cutoff was required for this gene. The whole procedure lasest $90 \pm 10 \mathrm{~min}$.

\section{External quality controls}

To verify the quality and reproducibility of the different RT-PCR analyses obtained with the Idylla platform, frozen samples $(500 \mu \mathrm{L}$ each) from 16 COVID-19 positive and 9 COVID-19 negative patients analysed at the COVID-19 Biobank of Nice were sent to the Department of Public Health in Naples (Italy) to be processed independently on another Idylla platform using the IdyllaTM SARS-CoV-2 CE-IVD Test. Among the selected positive SARS-CoV-2 samples, 8 showed an elevated CT value (over $30 \mathrm{CT}$ ) and the 8 others a low CT value (under $20 \mathrm{CT}$ ). 


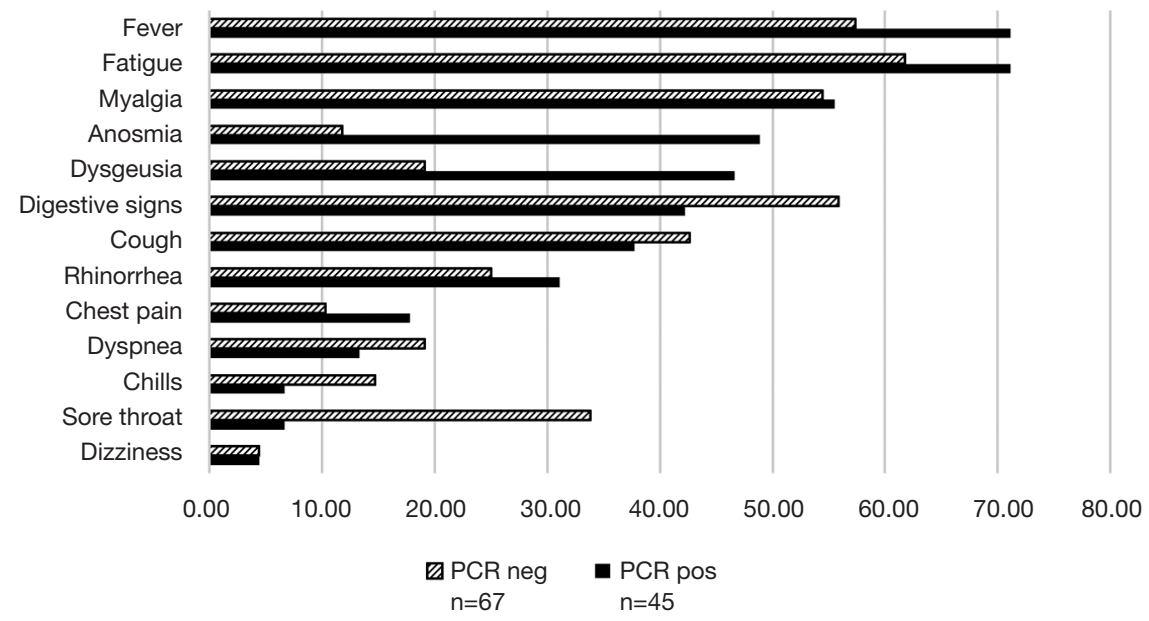

Figure 1 Self-reported symptoms (\%).

\section{Statistical analysis}

Continuous variables are presented as means $( \pm \mathrm{SD})$, and categorical variables as numbers and percentages. Baseline characteristics between patients with or without COVID-19 were compared using the Student $t$-test or Wilcoxon-Mann Whitney test for quantitative variables depending on the normality of distribution of the parameters, or the chisquare test for qualitative variables. Positive and negative agreement between the Biocartis test (index test) and the SOC tests (SYNLAB Barla or CHU of Nice reference laboratories) were evaluated. For each RNA target detected (the Orf1b and $\mathrm{N}$ targets) the Wilcoxon signed-rank test for comparing paired data was used to compare CTs obtained with the index test and CTs obtained with the SOC test. The Pearson correlation test was used to assess the dependency between CTs obtained with the index test and CTs obtained with the SOC care test. The IdyllaTM SARSCoV-2 Test was compared to the SOC reference method by calculating the percentage of concordance. Lastly, using the Idylla $^{\mathrm{TM}}$ data, the number of RNA targets for each positive result, depicted in percentage, was assessed.

\section{Ethics and regulatory clearances}

The study was conducted in accordance with the Declaration of Helsinki (as revised in 2013). Ethics committee approval (CPP Sud Méditerranée V; registration \# 20.04014.35208) was obtained on April 22, 2020. Liability Insurance: Hospital Mutual Insurance Company (SHAM $\mathrm{n}^{\circ}$ 159087). The study is registered at ClinicalTrial.gov,
NCT04418206. Informed consent was obtained from all individuals included in this study.

\section{Role of sponsor}

The funding organizations played no role in the design of the study, the choice of the patients, the collection, analysis or interpretation of data, the writing of the report nor in the decision to submit the paper for publication. The corresponding authors had full access to all of the data and the final responsibility to submit for publication.

\section{Results}

\section{Participants' characteristics}

One hundred and twelve subjects with mild COVID-19 symptoms underwent SARS-CoV-2 RT-PCR testing over the 13 -week study period (September 21 to December 19, 2020). One hundred and two patients were sampled at the downtown screening centre and 10 at the CHU of Nice screening centre. The interval between the onset of symptoms and testing was $3.8 \pm 2.7$ days and most of the participants [79/112 (70.5\%)] were sampled in the early stages of the disease (i.e., within 4 days of symptoms onset). Participants were predominantly women 69/113 (61.6\%). The mean $( \pm \mathrm{SD})$ age was $40 \pm 15$ years. Out of these 112 subjects, $45(40.2 \%)$ were diagnosed as positive for COVID-19. No clinical symptoms distinguished between SARS-CoV-2 RT-PCR positive and negative subjects (Figure 1), except for anosmia and dysgeusia which were 

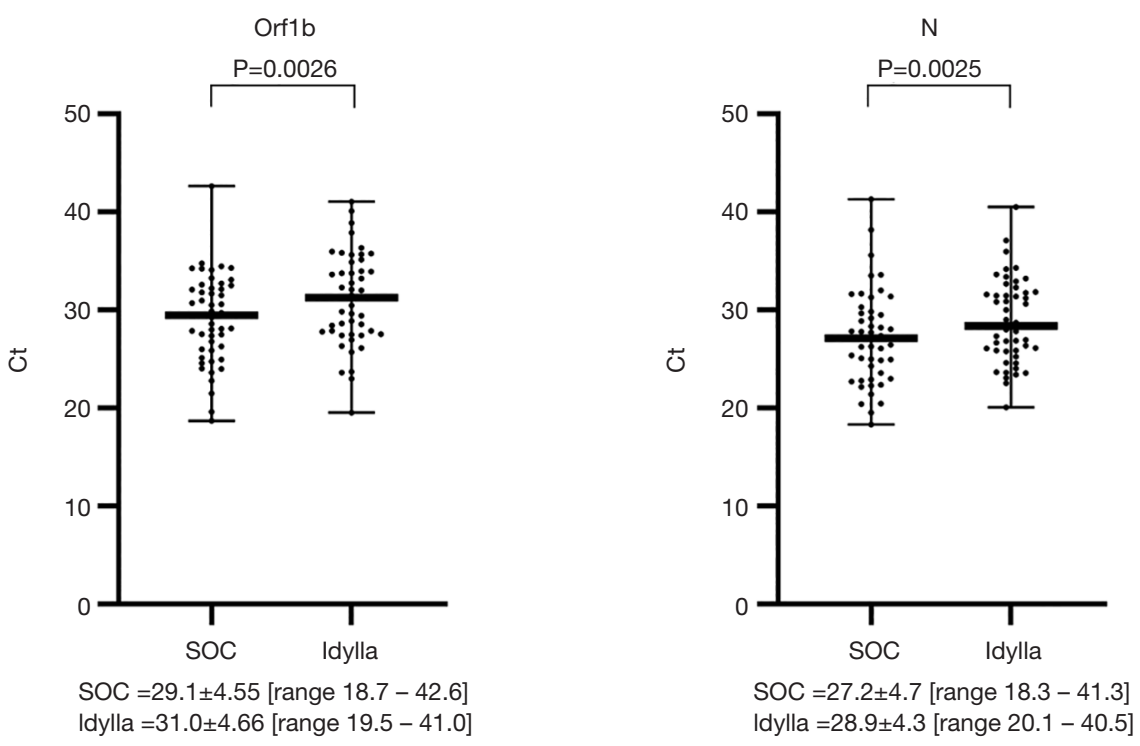

Figure 2 CT values for the Orf1b and N genes measured with the standard of care (SOC) comparator and the Idylla test.

significantly more frequent in SARS-CoV-2 PCR positive individuals $(\mathrm{n}=22 / 45,49 \%$ vs. $\mathrm{n}=8 / 67,12 \%, \mathrm{P}<0.001$ and $\mathrm{n}=21 / 45,47 \%$ vs. $\mathrm{n}=13 / 67,19 \% \mathrm{P}=0.008$ ). Sore throat was significantly more frequent in SARS-CoV-2 PCR negative individuals $(\mathrm{n}=23 / 67,34 \%$ vs. $\mathrm{n}=3 / 45,7 \%, \mathrm{P}<0.001)$. There was no significant difference between the oxygen saturation levels as measured by pulse oximetry between SARS-CoV-2 RT-PCR negative $\left(\mathrm{SpO}_{2}=98.3 \% \pm 2 \%\right)$ and SARS-CoV-2 RT-PCR positive subjects $\left(\mathrm{SpO}_{2}=97.7 \% \pm 2 \%\right)(\mathrm{P}=0.06)$.

\section{Performance of the IdyllaTM RT-PCR Test}

\section{Diagnostic accuracy}

Forty-five subjects out of 112 were diagnosed with COVID-19 based on early symptoms plus a positive RTPCR as determined by the reference laboratories. All these 45 subjects also had a positive RT-PCR with the Idylla system (positive agreement $100 \%$ ). Fifty-eight had a negative RT-PCR as determined by the reference laboratories; fifty-six of these had a negative RT-PCR with the Idylla system and two were positive with the Idylla RT-PCR test. These subjects had a positive COVID-19 serodiagnosis 21 days later. Thus, the Idylla SARS-CoV-2 RT-PCR results were classified as true positives and the comparative results of the SOC reference laboratory were classified as false negatives. In total, these results showed a sensitivity and a specificity of $100 \%$ for the Idylla system (negative agreement 100\%).
Relationship between the CT measured with the Idylla test and the SOC comparator

For both target genes, CT values with the Idylla test were significantly higher than those of the standard of care test (Figure 2). There was quite a weak but statistically significant correlation between the CT values obtained with the Idylla and SOC tests (Figure 3).

\section{External validation}

The comparative analyses (positive/negative) and the CT values obtained in the laboratories in Nice and Naples were processed as inter-laboratory controls. $100 \%$ concordance was observed for the positive and negative results obtained in Nice and Naples with non-significant variation concerning the CTs obtained for the Orf1b and $\mathrm{N}$ genes for the positive patients $(25.9 \pm 5$ and $29.1 \pm 4$ vs. $26.7 \pm 3$ and $30.2 \pm 2$ for Orf1b and $\mathrm{N}$ in Nice $v s$. Naples, respectively) (data not shown).

\section{Relationship between the CTs for the Orf1b and N genes with the Idylla and SOC tests}

The Idylla test showed a strong and significant correlation between $\mathrm{Cq}$ values obtained for the Orf1b and $\mathrm{N}$ genes while the SOC test gave a weaker but significant correlation between CT values obtained for the Orf1 b and $\mathrm{N}$ genes (Figure 4). Additionally, the majority ( $>95 \%$ ) of Idylla tests had all 5 targets detected but that indeed some rare cases will not have all 5 targets detected (data not shown). 

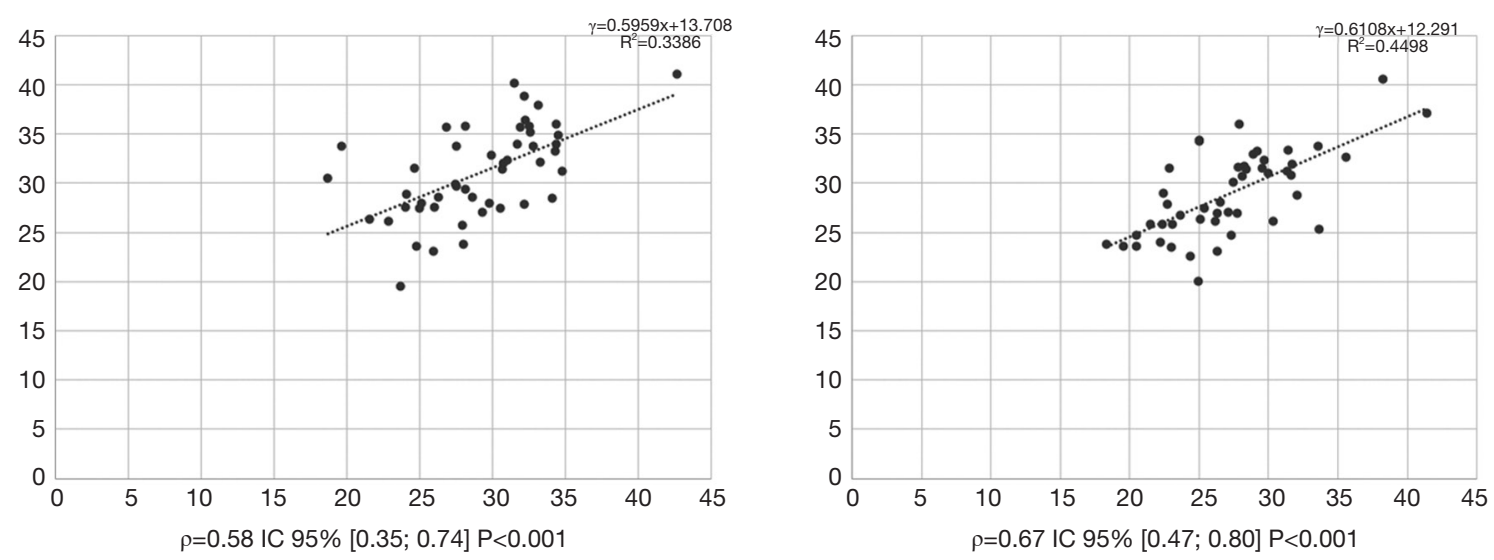

Figure 3 Relationship between CTs measured with the Idylla test ( $\mathrm{Y}$ axis) and the SOC comparator (X axis) for the Orf1b and N genes.
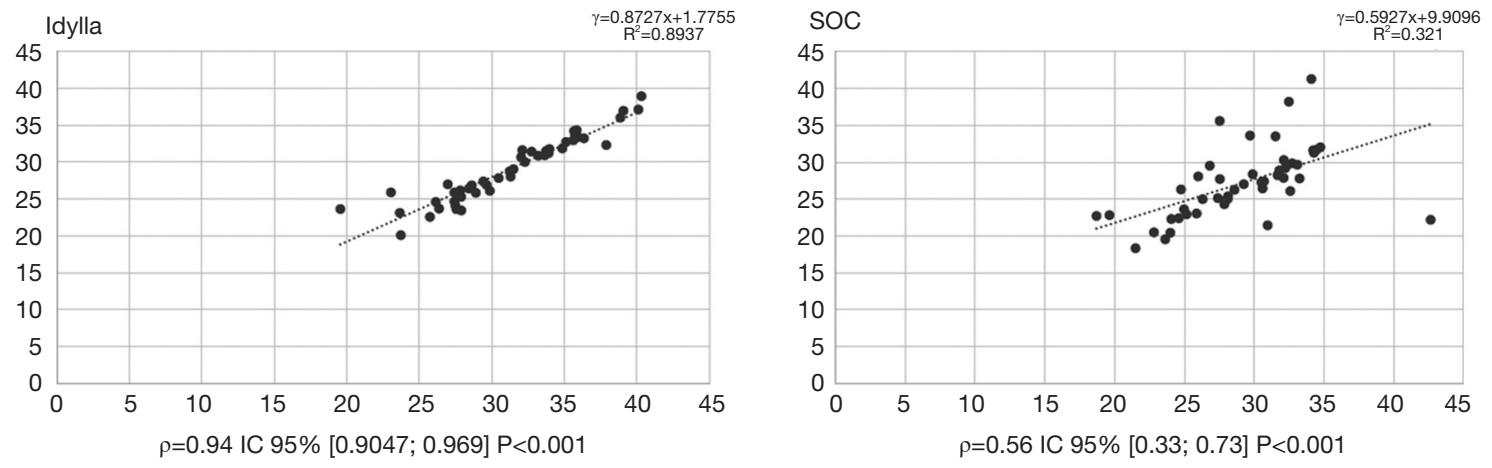

Figure 4 Relationship between the CTs measured for the Orf1b (X axis) and N genes ( $\mathrm{Y}$ axis) with the Idylla and standard of care tests.

\section{Discussion}

Our study demonstrated that a highly sensitive, specific and easy to use automatized RT-PCR test that provides a fast turnaround time for detection of SARS CoV-2, can be rapidly set up as a safe test, notably for urgent clinical needs. Since the beginning of the COVID-19 crisis, hundreds of tests and technologies have been or are currently being deployed for diagnosis of SARS-CoV-2 infections, albeit many still await clinical validation (5-8) Most of them are automatized options but performed after a mandatory step of nucleic acids extraction from fresh cytological or fluid samples $(5,9,10)$. Therefore, the majority of the so far available methods require handling of fresh samples for nucleic acids extraction in a BSL-2 environment for biohazard protection of $\mathrm{HCW}$, according to the international guidelines for laboratory tests performed during the COVID-19 pandemic $(7,11)$. Recently, Gibani et al. developed a promising method called "CovidNudge", which could be of strong interest for point-of-care testing (12). Indeed, this technology did not require separate nucleic acid extraction and amplification steps. Interest in the use of this type of technology as well as that of the Idylla technology used in the present work are certainly the first to strongly limit the possibility of cross contamination during notably the pre-analytical phases.

For most of the current RT-PCR methods used for SARS-CoV-2 detection, the results are usually obtained after an overall time of an average of 6 hours between the reception of the sample, the sequencing results and the validation of the report by the biologist. But sometimes a longer delay of even upto 24 hours occurs. Importantly, the different pre-analytical and analytical phases need to be mastered by qualified technicians with expert training in molecular biology. Moreover, the results need to be qualified by the biologists for validation before transmission 
to the physician.

Furthermore, several RT-PCR approaches need to run several samples at the same time for analyses to optimize the use of the same batch of reagents. Thus, it is quite difficult to run one by one the samples on reception in the biology laboratory, which hampers go with the flow. This may potentially hinder the workflow of the samples and therefore induce a delay in obtaining the results, notably in some urgent clinical settings.

The Idylla test used in this study can be performed within 90 minutes without any risk of contagion due to sample handling and could be done, if necessary, outside a BSL-2 environment in the same way the rapid antigenic test is done for SARS-Cov-2 detection $(13,14)$. It is mandatory that the HCWs follow the international guidelines concerning the wear of personnel protection equipment $(2,15)$. The results and complete reports can be transmitted immediately in a safe manner to the physicians at the clinical department or even at home via internet from the computer linked to different devices.

The results of the present study demonstrated 100\% sensitivity and specificity with the Idylla SARS-CoV-2 Test compared with two of the defined SOC tests used in the Nice area. Moreover, cross validation between two laboratories (CHU of Nice and the Naples Hospital) of Idylla platforms showed high concordance with negative and positive SARS-CoV-2 samples (16-19).

A certain number of pitfalls exist when doing molecular biology tests for detection of SARS-CoV-2. It is important to be aware of the occurrence of potential false negative results, notably using RT-PCR approaches (20). Interestingly, in our study the three comparative tests did not have the exact same gene target design. However, despite the different designs we obtained a high level of concordance for these comparative tests. Moreover, when evaluating the viral load, which can be indirectly estimated from the different CT values, the results showed relatively good concordance between the different tests. This information may be of considerable interest to the physician since the severity and the prognosis of the disease may correlate with the viral load, independently of the associated risk factors (10).

Recently, many tests have been developed and optimized, including RT-LAMP, which can detect the SARS-CoV-2 under 60 minutes or even in less than 30 minutes (21). These tests could be an alternative approach to the RT-PCR approach for rapid assessment of the COVID-19 status (21). The RT-LAMP technique amplifies the nucleic acid in one step using reverse transcriptase, DNA polymerase and four to six oligonucleotides of a certain architecture. There are a number of advantages of the RT-LAMP approach for the detection of the SARS-CoV-2 virus. First, the detection time of SARS-CoV-2 by RT-LAMP is shorter compared to the current RT-PCR tests. According to Jiang et al., the process time to detect SARS-CoV-2 using RT-LAMP is two times faster than that for qRT-PCR (22) However, despite many benefits, LAMP techniques have several limitations. First, these techniques are mainly associated with a low sensitivity of single tests and the optimization of primer design and reaction conditions. In this regard, for a RTLAMP test with RNA extraction, a study found that the sensitivity of RT-LAMP was $75 \%$ despite the absence of false positive results (23). The sensitivity was $86.4 \%$ when the RT-PCR was used to identify the E gene (23). The second disadvantage of RT-LAMP is that the process for designing primers and probes capable of targeting specific gene sequences can be complex (24). As a result, primer optimization might be limited. Thirdly, RT-LAMP has only been able to detect SARS-CoV-2 in patients with a high viral load. Hence, a low RNA input might lead to false negative results. In a study by Jiang et al. into primer design for LAMP techniques, the virus could not be detected in four positive patients when the volume of RNA input was 2 microliters (22). This amount was 2.5 times less than the amount of RNA used for qRT-PCR.

Antigen-detecting rapid diagnostic tests (Ag-RDTs) using NPS are highly specific and allow a quick and simple diagnosis in patients with early symptoms. The trade-off for simplicity and speed of operation of Ag-RDTs is a decrease in sensitivity compared to RT-PCR tests and none of the antigenic tests evaluated so far meet the requirements of diagnostic performance, in terms of sensitivity, allowing use as an alternative to PCR for the diagnosis of COVID-19 in symptomatic subjects (overall sensitivity of around $60 \%$ compared to PCR for the best tests) (14). However, even if rapid testing is critical for COVID-19 diagnosis, we need to be aware of the sensitivity of the different tests available nowadays. We strongly believe that the Idylla SARS-CoV-2 Test presents many advantages considering not only its very high sensitivity and specificity, but also the reasonable turnaround time, in less than 90 minutes, to obtain very reliable results while using a rather convenient technique. It is noteworthy that given the PCR design, the Idylla test for SARS CoV-2 detection is not affected by the new UK and South African variants and will not give false negative results with cases infected with these new variants 
(communication manufacturer Biocartis).

In conclusion, the present study conducted in a prospective cohort of subjects suspected of COVID-19 demonstrated that a fast, highly sensitive and specific RTPCR test can be easily set up on an Idylla platform, enabling forthright decision making and patient addressing into different clinical departments. However, we need to keep in mind that this new test cannot replace the current molecular tests developed in most of the biological laboratories for SARS-CoV-2 detection, since no more than eight patients could be analysed at the same time at the Nice Hospital with a 90 minutes average response time. Therefore, depending on the urgency of the clinical situation an Idylla analysis can be proposed by the physician.

\section{Acknowledgments}

Funding: This work received organizational and financial support from the Conseil Départemental 06 and the Métropole Nice Côte d'Azur.

\section{Footnote}

Reporting Checklist: The authors have completed the MDAR reporting checklist. Available at http://dx.doi.org/10.21037/ atm-21-690

Data Sharing Statement: Available at http://dx.doi. org/10.21037/atm-21-690

Conflicts of Interest: On submission of the manuscript, all authors completed the ICMJE uniform disclosure form (available at http://dx.doi.org/10.21037/atm-21-690). PH is a member of the scientific advisory board of Biocartis (Mechelen, Belgium). The other authors have no conflicts of interest to declare.

Ethical Statement: The authors are accountable for all aspects of the work in ensuring that questions related to the accuracy or integrity of any part of the work are appropriately investigated and resolved. Research involving human subjects complied with all relevant national regulations, institutional policies and is in accordance with the tenets of the Helsinki Declaration (as revised in 2013), and has been approved by the authors' Institutional Review Board (CPP Sud Méditerranée V; registration \# 20.04014.35208). Informed consent was obtained from all individuals included in this study.
Open Access Statement: This is an Open Access article distributed in accordance with the Creative Commons Attribution-NonCommercial-NoDerivs 4.0 International License (CC BY-NC-ND 4.0), which permits the noncommercial replication and distribution of the article with the strict proviso that no changes or edits are made and the original work is properly cited (including links to both the formal publication through the relevant DOI and the license). See: https://creativecommons.org/licenses/by-nc-nd/4.0/.

\section{References}

1. Hanson KE, Caliendo AM, Arias CA, et al. Infectious Diseases Society of America Guidelines on the Diagnosis of COVID-19. Clin Infect Dis 2020. [Epub ahead of print]. doi: 10.1093/cid/ciaa760.

2. Lippi G, Adeli K, Ferrari M, et al. Biosafety measures for preventing infection from COVID-19 in clinical laboratories: IFCC Taskforce Recommendations. Clin Chem Lab Med 2020;58:1053-62.

3. Lechien JR, Chiesa-Estomba CM, Place S, et al. Clinical and epidemiological characteristics of 1420 European patients with mild-to-moderate coronavirus disease 2019. J Intern Med 2020;288:335-44.

4. Tanga V, Leroy S, Fayada J, et al. Establishment of a Collection of Blood-Derived Products from COVID-19 Patients for Translational Research: Experience of the LPCE Biobank (Nice, France). Biopreserv Biobank 2020;18:517-24.

5. Li C, Zhao C, Bao J, et al. Laboratory diagnosis of coronavirus disease-2019 (COVID-19). Clin Chim Acta 2020;510:35-46.

6. Lippi G, Horvath AR, Adeli K. Editorial and Executive Summary: IFCC Interim Guidelines on Clinical Laboratory testing during the COVID-19 Pandemic. Clin Chem Lab Med 2020;58:1965-9.

7. Thompson S, Bohn MK, Mancini N, et al. IFCC interim guidelines on biochemical/hematological monitoring of COVID-19 patients. Clin Chem Lab Med 2020;58:2009-16.

8. Vandenberg O, Martiny D, Rochas O, et al. Considerations for diagnostic COVID-19 tests. Nat Rev Microbiol 2021;19:171-83.

9. Poljak M, Korva M, Gašper NK. Clinical Evaluation of the cobas SARS-CoV-2 Test and a Diagnostic Platform Switch during 48 Hours in the Midst of the COVID-19 Pandemic. J Clin Microbiol 2020;58:e00599-20.

10. Pujadas E, Ibeh N, Hernandez MM, et al. Comparison 
of SARS-CoV-2 detection from nasopharyngeal swab samples by the Roche cobas 6800 SARS-CoV-2 test and a laboratory-developed real-time RT-PCR test. J Med Virol 2020;92:1695-8.

11. Loh TP, Horvath AR, Wang CB, et al. Laboratory practices to mitigate biohazard risks during the COVID-19 outbreak: an IFCC global survey. Clinical Chemistry and Laboratory Medicine (CCLM). Clin Chem Lab Med 2020;58:1433-40.

12. Gibani MM, Toumazou C, Sohbati M, et al. Assessing a novel, lab-free, point-of-care test for SARS-CoV-2 (CovidNudge): a diagnostic accuracy study. Lancet Microbe 2020;1:e300-7.

13. Agence ontarienne de protection et de promotion de la santé (Santé publique Ontario). Aspects de biosécurité à considérer en lien avec le test rapide antigénique PanbioMCCOVID-19 d'Abbott. Toronto, ON. Imprimeur de la Reine pour l'Ontario, 2020. Available online: https:// www.publichealthontario.ca/-/media/documents/lab/ covid-19-abbott-panbio-antigen-rapid-test-biosafety. pdf?la=fr. :2.

14. Organization $W H$. Antigen-detection in the diagnosis of SARS-CoV-2 infection using rapid immunoassays: interim guidance, 11 September 2020. World Health Organization 2020 [cited 2021 Jan 23]; Available online: https://apps. who.int/iris/handle/10665/334253

15. Hofman P. Challenges and issues surrounding the use for translational research of human samples obtained during the COVID-19 pandemic from lung cancer patients. Transl Lung Cancer Res 2020;9:1543-53.

16. Luca CD, Sgariglia R, Nacchio M, et al. Rapid On-site Molecular Evaluation in thyroid cytopathology: A sameday cytological and molecular diagnosis. Diagnostic

Cite this article as: Hofman P, Boutros J, Benchetrit D, Benzaquen J, Leroy S, Tanga V, Bordone O, Allégra M, Lespinet V, Fayada J, Maniel C, Griffonnet J, Selva E, Troncone G, Portella G, Lavrut T, Chemla R, Carles M, Ilié M, Marquette C. A rapid near-patient RT-PCR test for suspected COVID-19: a study of the diagnostic accuracy. Ann Transl Med 2021;9(11):921. doi: 10.21037/atm-21-690
Cytopathology 2020;48:300-7.

17. Uguen A, Troncone G. A review on the Idylla platform: towards the assessment of actionable genomic alterations in one day. J Clin Pathol 2018;71:757-62.

18. Heeke S, Hofman P. EGFR Mutation Analysis in Nonsmall Cell Lung Carcinoma from Tissue Samples Using the Fully Automated IdyllaTM qPCR System. Methods Mol Biol 2019;2054:147-55.

19. Lassalle S, Hofman V, Heeke S, et al. Targeted Assessment of the EGFR Status as Reflex Testing in Treatment-Naive Non-Squamous Cell Lung Carcinoma Patients: A Single Laboratory Experience (LPCE, Nice, France). Cancers (Basel) 2020;12:95.

20. Wernike K, Keller M, Conraths FJ, et al. Pitfalls in SARS-CoV-2 PCR diagnostics. Transbound Emerg Dis 2021;68:253-7.

21. Thompson D, Lei Y. Mini review: Recent progress in RT-LAMP enabled COVID-19 detection. Sensors and Actuators Report 2020;2:100017.

22. Jiang M, Pan W, Arasthfer A, et al. Development and Validation of a Rapid, Single-Step Reverse Transcriptase Loop-Mediated Isothermal Amplification (RT-LAMP) System Potentially to Be Used for Reliable and HighThroughput Screening of COVID-19. Front Cell Infect Microbiol 2020;10:331.

23. Rödel J, Egerer R, Suleyman A, et al. Use of the variplexTM SARS-CoV-2 RT-LAMP as a rapid molecular assay to complement RT-PCR for COVID-19 diagnosis. J Clin Virol 2020;132:104616.

24. Kashir J, Yaqinuddin A. Loop mediated isothermal amplification (LAMP) assays as a rapid diagnostic for COVID-19. Med Hypotheses 2020;141:109786. 


\section{Supplementary}

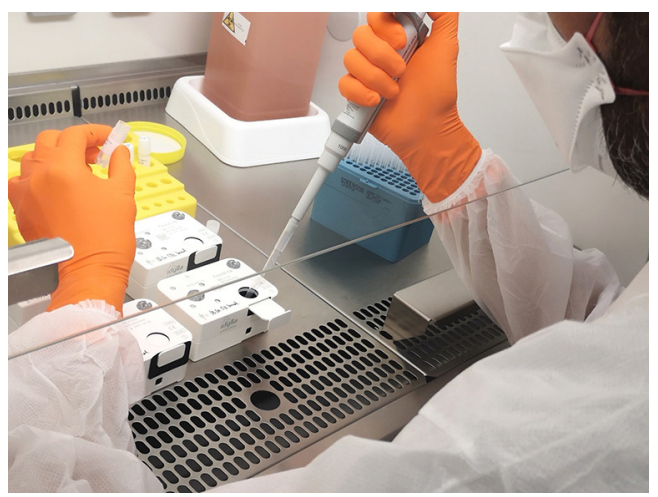

Figure S1 Sample loading into the Idylla cartridge under a BSC-2 hood.

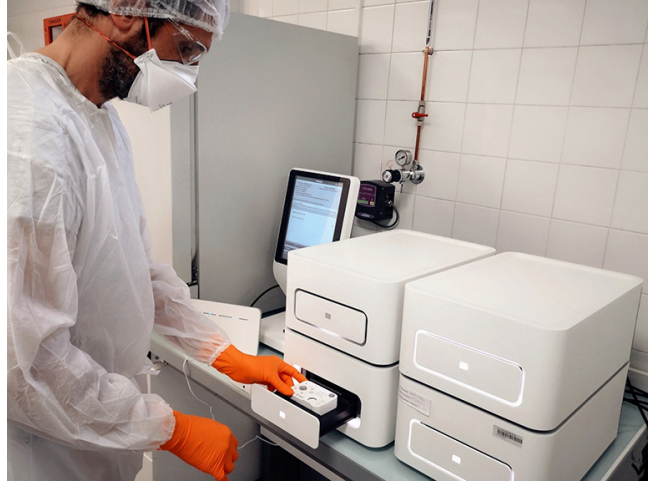

Figure S2 Four consoles of Idylla used in the COVID-19 biobank of the Nice University Hospital. 